\title{
Endovascular catheter knot untying: A new technique
}

\author{
Erik Soule, Mario Agrait, Megan Deacon-Casey, Travis Meyer, Jesse Martin, Jerry Matteo*
}

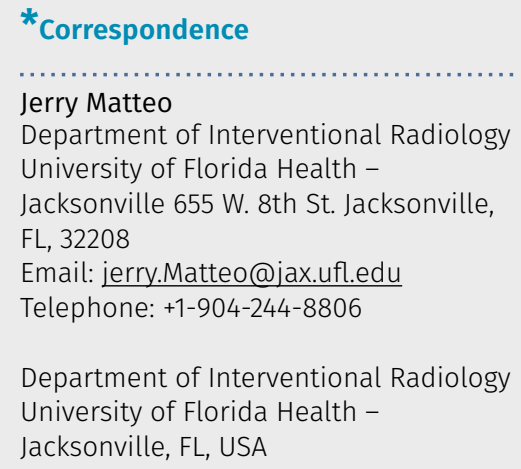

\begin{abstract}
Indwelling vascular catheters may be maintained for extended periods of time especially for critically ill or hospitalized patients requiring frequent blood draws. If they become knotted within the vasculature, serious iatrogenic sequelae can result, such as: pneumothorax, atrial arrhythmias, ventricular arrhythmias, pulmonary embolism, pulmonary hemorrhage, intracardiac rupture, pulmonary artery rupture, balloon rupture, bacteremia, and death. surgical cut-down may be indicated for removal of knots that preclude catheter retrieval and are refractory to intravascular removal modalities. Three such knots were untied using the intraluminal technique described in this report. The technique utilizes balloon angioplasty in conjunction with a wire to safely and effectively untie knotted catheters.
\end{abstract}

Keywords: Vascular catheters, Arrhythmias, Angioplasty balloon.

\section{Introduction}

Intravascular knot formation was first described by Johansson in 1954 [1]. Often going unrecognized until it is time to remove the device, intravascular knots can preclude retrieval of indwelling catheters. Both surgical and non-surgical methods have been described for the removal of knotted catheters and wires [2]. Devices known to form intravascular knots include cardiac catheters, central venous catheters, arteriography catheters, carotid arteriotomy guidewires, pacemaker electrodes, and pulmonary artery catheters. Risk factors for intravascular knot formation include increased catheter length, pliability, decreased catheter wall thickness, and placement without fluoroscopy [3].

Surgical removal of knotted catheters and wires is considered the last resort. Such surgical approaches are usually reserved for complex multiple looped wires. Several minimally invasive methods have been developed to address this problem. Examples include tightening the knot to decrease the diameter enough for safe removal through the vein insertion, use of large introducer sheaths, dilators, tip deflecting wires, angioplasty balloons in conjunction with snare devices, biopsy forceps, and the use of sheaths and parallel crossing wires to remove or untangle knots [4-7]. This report describes a technique utilizing an angioplasty balloon to untie such knots. This technique has been employed to safely and effectively untie and remove three separate knotted catheters.

The Swan-Ganz (SG) pulmonary artery catheter was first developed by Dr. Jeremy Swan and Dr. William Ganz in 1970 as a way of measuring left atrial pressure. Historically, SG catheters saw widespread use especially in the intensive care (ICU) setting. In 2006 it was estimated that over 1.2 million SG catheters were placed each year in the United States [8]. The incidence of serious complications associated with the use of SG catheters has been reported to be between $3-17 \%$ percent [9]. It is estimated that more than two-thirds of all intravascular knots are caused by pulmonary artery catheters [10]. Today, SG catheters are used especially in critically ill patients and those undergoing cardiothoracic surgery. 


\section{Technical Report}

An 80 year old man with history of severe COPD, was unable to be extubated following surgery and was admitted to the ICU where he received mechanical ventilation and placement of an SG catheter within the right subclavian vein. As the patient recovered, the SG catheter was no longer necessary for invasive hemodynamic monitoring. Repeated attempts were made to remove the catheter, but only approximately $20 \mathrm{~cm}$ could be withdrawn. A frontal radiograph was obtained revealing a knot in the distal portion of the catheter within the left pulmonary artery (Figure 1). Interventional radiology was subsequently consulted for catheter removal. The right neck and upper chest were prepared surrounding the existing SG catheter and draped in the usual sterile fashion after informed consent was obtained. A 0.035-inch stiff angled hydrophilic wire (Glidewire; Terumo Medical, Somerset, NJ) was introduced via the lumen of the SG catheter as a primary attempt at knot loosening, which was unsuccessful despite multiple attempts. Using manual traction, the catheter was positioned into the superior vena cava without complication (Figure 2). The knot was recalcitrant to all techniques employed to untie or loosen it utilizing the existing subclavian vein access.

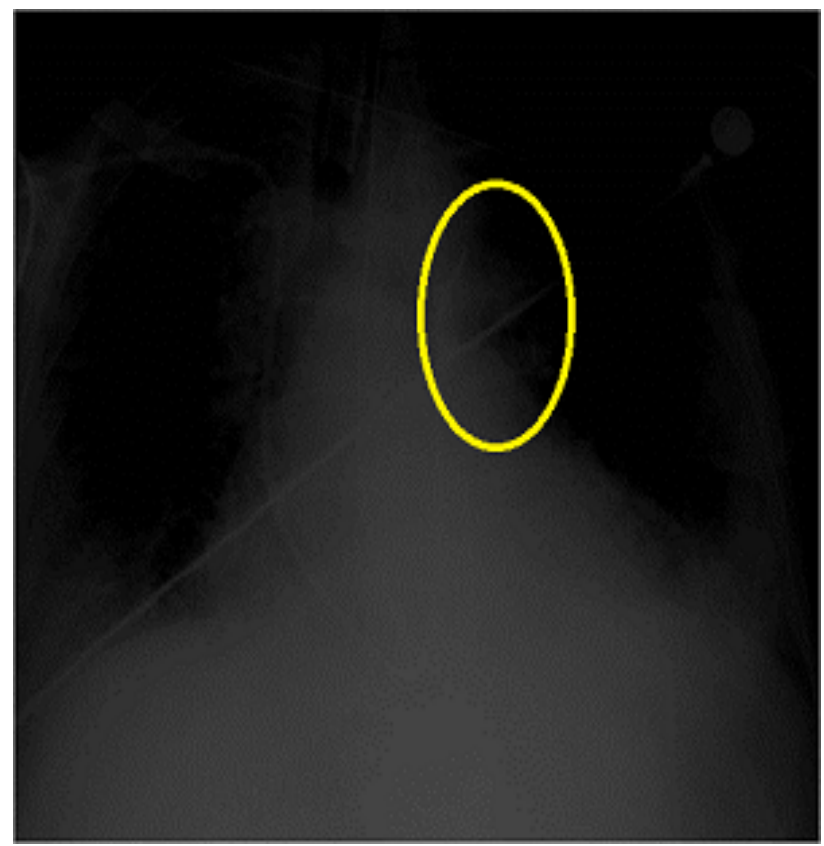

Figure 1: Single frontal chest radiograph revealing a knotted Swan-Ganz catheter (yellow circle) in the left pulmonary artery with the image purposely windowed to show the catheter.

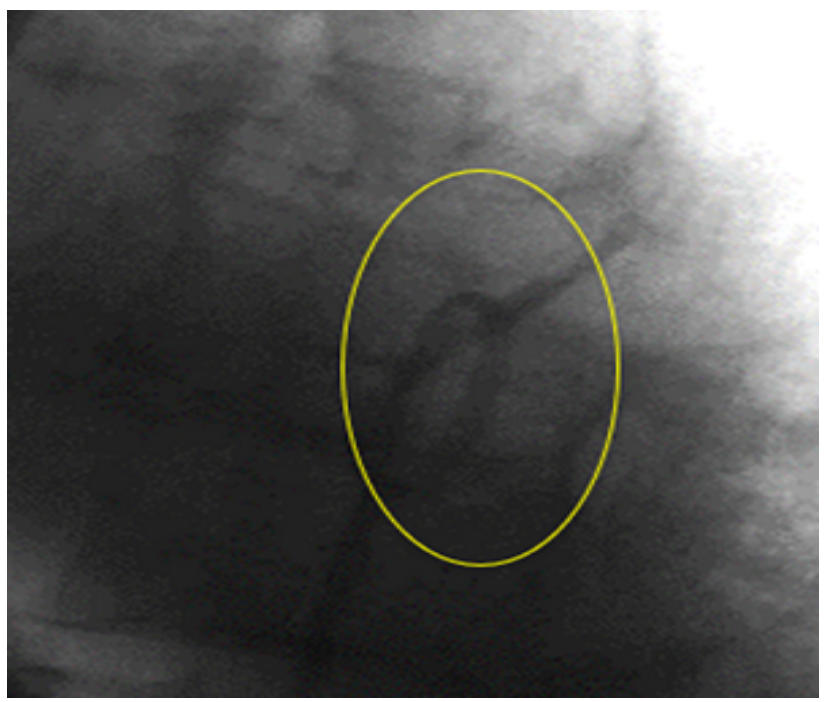

Figure 2: Fluoroscopic magnified image of the knot in the SwanGanz catheter (yellow circle) after it was retracted and positioned in the SVC.

The decision was then made to attempt intravascular unknotting via a groin approach. The right groin was prepared and draped in the usual sterile fashion. The right common femoral vein was punctured with a micropuncture set. This access site was dilated to accept a 7-French introducer sheath which was positioned in the right common femoral vein. Via the sheath, a 5-French angled hydrophilic catheter (Glidecath; Terumo Medical, Somerset, NJ) and Glidewire combination were advanced and guided through the center of the knot in the SG catheter within the SVC (Figure 3). The Glidecath was then exchanged for a $14 \mathrm{~mm} \times 40 \mathrm{~mm}$ percutaneous transluminal angioplasty balloon and inflated maximally (Figure 4). This maneuver loosened and partially opened the knot. The knot was then fully untied by reintroducing the Glidewire into the SG catheter through the subclavian vein access.

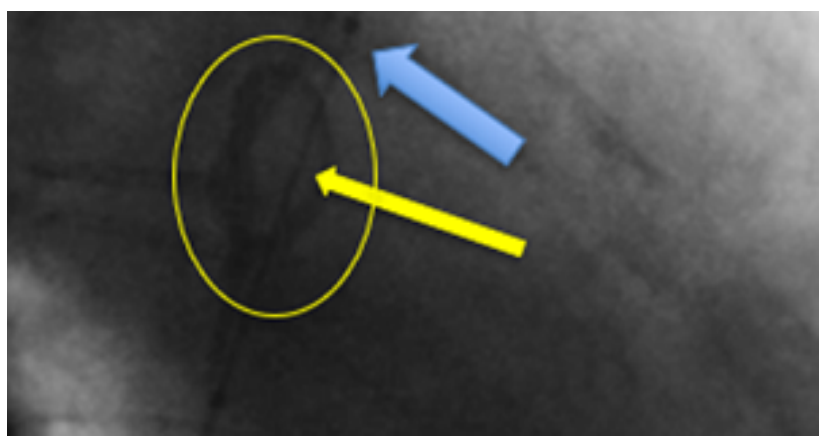

Figure 3: 5-French angled hydrophilic catheter (blue arrow) over a stiff angled hydrophilic wire (yellow arrow) are guided into the center of the knot (yellow circle). 


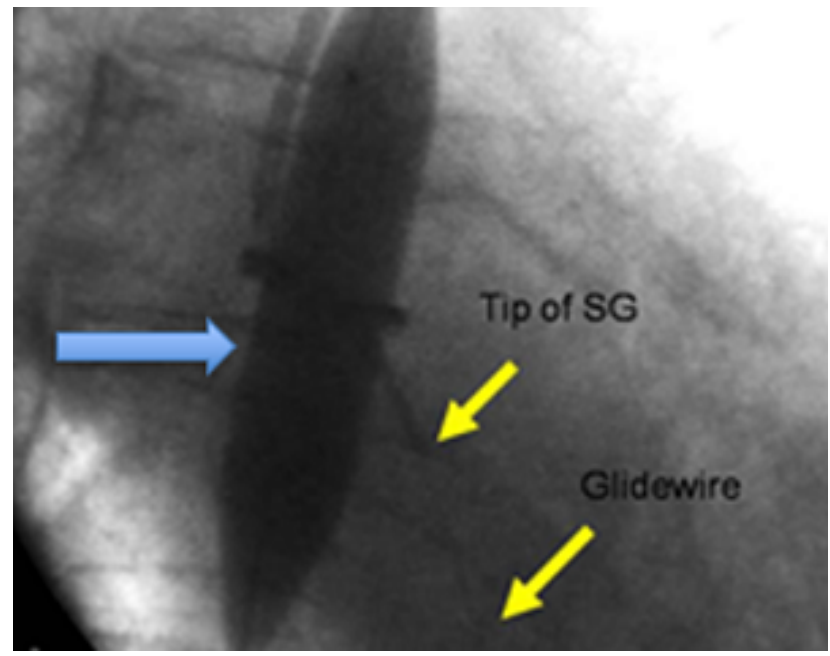

Figure 4: Angioplasty balloon (blue arrow) fully inflated over the glidewire to loosen the knot within the Swan-Ganz Catheter.

After the knot was untied intravascularly, the SG catheter was withdrawn in its entirety without incident from the right subclavian vein (Figure 5). All vascular devices were removed and access site hemostasis was achieved by using manual compression. No procedure related complications were observed.

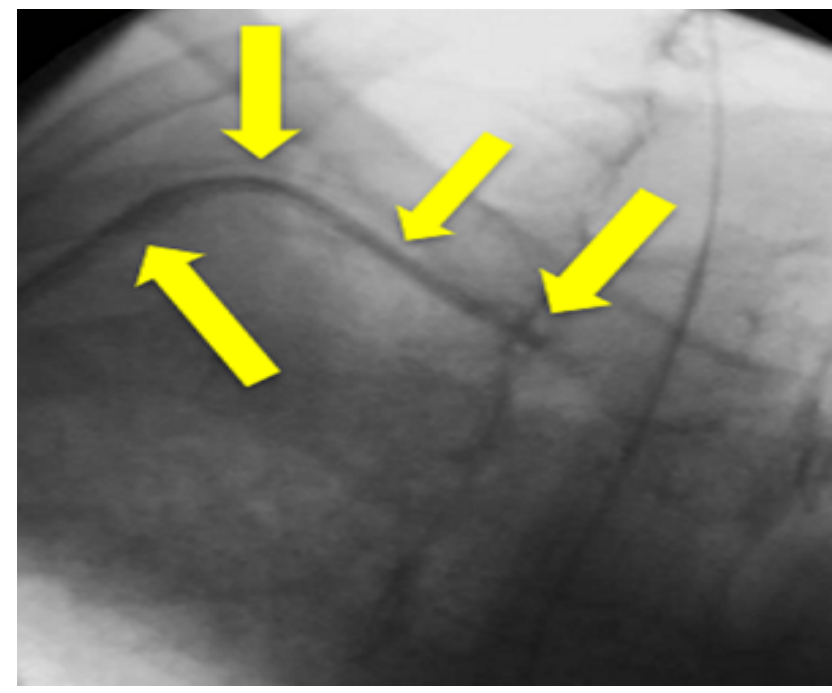

Figure 5: Fluoroscopic image revealing Swan-Ganz catheter from a right internal jugular approach fully untied, which was easily removed in its entirety (yellow arrows).

\section{Discussion}

Knot formation is an uncommon complication of indwelling catheters including those in the pulmonary arteries and may preclude safe and straightforward catheter removal. Serious complications of SG insertion occur with an incidence of $3-17 \%$ and may be catastrophic. Knot formation is a well-recognized complication of pulmonary artery catheterization that may preclude safe bedside catheter removal. The most common location for SG knot formation is in the cardiac chambers. This occurs during the placement process as the catheter deflects off the wall of the cardiac chamber and folds back on itself forming a knot [11]. Risk of intravascular knot formation increases when placement is performed without the use of fluoroscopy. The majority of intravascular knots can be unraveled by simple maneuvering, however, complex knots may necessitate a more creative approach [12].

Although surgery has been employed for knotted pulmonary artery catheter removal, minimally invasive techniques have become the mainstay as they allow for increased patient comfort and safety. The technique described in this report utilizes a balloon angioplasty catheter in conjunction with wire techniques to safely remove a knotted catheter. This method may be applied to any knotted catheter and thus increases the interventional radiologist's repertoire for addressing potentially devastating iatrogenic indwelling catheter knots.

\section{Conclusion}

Intravascular interventional techniques are the primary modality to remove knotted catheters. Several techniques are available, however the method described in this report is safe, quick, and effective and uses standard available equipment. Three knotted SG catheters were successfully untied and removed using an angioplasty balloon and this endovascular strategy.

\section{Conflict of interest}

The authors declare no conflicts of interest.

\section{Copyrights}

(c) Erik Soule et al. 2018; licensee OA Journal of Radiology. This is an Open Access article distributed under the terms of the Creative Commons Attribution License (http:// creativecommons.org/licenses/by/4.0), which permits unrestricted use, distribution, and reproduction in any medium, provided the original work is properly credited.

\section{References}

1. Johansson L, Malmstrom G, Ugglia LG. (1954) Intracardiac knotting of the catheter in heart catheterization. J Thorac Surg. 27: 605-607.

2. Georghiou GP, Vidne BA, Raanani E. (2004) Knotting of a pulmonary artery catheter in the superior vena cava: surgical removal and a word of caution. Heart. 90: e28. 
3. Ranatunga DG, Richardson MG, Brooks DM. (2007) Percutaneous fluoroscopic removal of a knotted Swan-Ganz catheter in a patient with a persistent left-sided superior vena cava. Australas Radiol. 51: 182-185.

4. Kumar SP, Yans J, Kwatra M, et al. (1980) Removal of a knotted flow-directed catheter by a nonsurgical method. Ann Intern Med. 92: 639-640.

5. Dach JL, Galbut DL, LePage JR. (1981) The knotted Swan-Ganz catheter: new solution to a vexing problem. AJR Am J Roentgenol. 137: 1274-1275.

6. Alzen G, Wildberger J, Klinge U, et al. (1991) Transfemoral extraction of a knotted Swan-Ganz catheter using an F24 dilator]. Anasthesiol Intensivmed Notfallmed Schmerzther. 26: 280-282.

7. Bhatti WA, Sinha S, Rowlands P. (2000) Percutaneous untying of a knot in a retained Swan-Ganz catheter. Cardiovasc Intervent Radiol. 23: 224-225.
8. Hadian M, Pinsky MR. (2006) Evidence-based review of the use of the pulmonary artery catheter: impact data and complications. Crit Care. 3: S8.Boyd KD, Thomas SJ, Gold J, et al. (1983) A prospective study of complications of pulmonary artery catheterizations in 500 consecutive patients. Chest. 84: 245-249.

9. Karanikas ID, Polychronidis A, Vrachatis A, et al. (2002) Removal of knotted intravascular devices. Case report and review of the literature. Eur J Vasc Endovasc Surg. 23: 189-194.

10. Ismail KM, Deckmyn TJ, Vandermeersch E, et al. (1998) Nonsurgical extraction of intracardiac double-knotted pulmonary artery catheter. J Clin Anesth. 10: 160-162.

11. Valenzuela-García LF, Almendro-Delia M, González-Valdayo M, et al. (2007) Percutaneous retrieval of a pulmonary artery catheter knot in pacing electrodes. Cardiovasc Intervent Radiol. 30: 1082-1084. 